\title{
Review
}

\section{A continued saga of Boc5, the first non-peptidic glucagon-like peptide-1 receptor agonist with in vivo activities}

\author{
Min $\mathrm{HE}^{1,2}$, Ni GUAN², Wei-wei GAO ${ }^{1}$, Qing LIU ${ }^{1,2}$, Xiao-yan WU ${ }^{1}$, Da-wei MA ${ }^{3}$, Da-fang ZHONG ${ }^{2}$, Guang-bo GE ${ }^{4}$, Chuan $\mathrm{LI}^{2}$, \\ Xiao-yan CHEN ${ }^{2}$, Ling YANG ${ }^{4}$, Jia-yu LIAO $^{1,5}$, Ming-wei WANG ${ }^{1,2, *}$ \\ ${ }^{1}$ The National Center for Drug Screening and ${ }^{2}$ the State Key Laboratory of Drug Research, Shanghai Institute of Materia Medica, \\ Chinese Academy of Sciences, Shanghai 201203, China; ${ }^{3}$ Shanghai Institute of Organic Chemistry, Chinese Academy of Sciences, \\ Shanghai 200032, China; ${ }^{4}$ Dalian Institute of Chemical Physics, Chinese Academy of Sciences, Dalian 116023, China; ${ }^{5}$ Department of \\ Bioengineering, University of California, Riverside, California, CA 92521, USA
}

Glucagon-like peptide-1 (GLP-1)-based therapy presents a promising option for treating type 2 diabetes. However, there are several limitations relative to the peptidic GLP-1 mimetics currently on the market or under development. This concern has led to a continued interest in the search for non-peptidic agonists for GLP-1 receptor (GLP-1R). Here, we briefly review the discovery, characterization and current status of a novel class of cyclobutane-derivative-based non-peptidic agonists for GLP-1R, including Boc5 and its newly discovered analogue WB4-24. Although the oral bioavailability of such compounds still poses great challenges, the progress made so far encourages us to identify a truly ‘druggable’ small molecule agonist for GLP-1R.

Keywords: type 2 diabetes; glucagon-like peptide-1; non-peptidic agonist; Boc5; G-protein coupled receptor

Acta Pharmacologica Sinica (2012) 33: 148-154; doi: 10.1038/aps.2011.169

\section{Introduction}

Type 2 diabetes mellitus (T2DM) comprises an array of dysfunctions resulting from a combination of resistance to insulin action and inadequate insulin secretion. It is characterized by hyperglycemia and associated with microvascular, macrovascular and neuropathic complications. T2DM is also linked causally with obesity, which is a global health burden in itself ${ }^{[1]}$. Unless appropriate action is taken, it is predicted that there will be at least 350 million people in the world with T2DM by the year $2030^{[2]}$. This situation is further worsened by the fact that currently available drugs such as insulin, sulfonylureas and thiazolidinediones only have limited efficacy and do not address two major problems, declining $\beta$-cell function and associated obesity. Therefore, the quest for novel therapeutics to combat diabetes and related metabolic abnormalities is justifiably intensive.

Glucagon-like peptide-1 (GLP-1)-based therapy presents an alternative and effective approach to treat T2DM ${ }^{[3]}$. GLP-1 is a gastrointestinal peptide produced by the proglucagon gene

\footnotetext{
* To whom correspondence should be addressed. E-mail wangmw@mail.shcnc.ac.cn

Received 2011-09-08 Accepted 2011-11-12
}

in L-cells of the small intestine in response to nutrients. It is thought to exert anti-hyperglycemic effects by stimulating insulin secretion, slowing gastric emptying, inhibiting glucagon release, stimulating $\beta$-cell proliferation and differentiation, and improving satiety ${ }^{[4]}$. GLP-1 appears to be an ideal and complementary approach to T2DM management for several reasons ${ }^{[3]}$ : (i) glucose-dependent release of insulin does not cause hypoglycemia; (ii) suppression of food consumption leads to reduced body weight; and (iii) protection of $\beta$-cell mass and function lessens the severity of T2DM. However, a major limitation in extending these advantages to clinical benefits resides in the very short half-life of GLP-1 in vivo (approximately 1-2 $\mathrm{min})^{[5]}$. This is attributed to $\mathrm{NH}_{2}$-terminal degradation by dipeptidyl peptidase IV (DPP-IV) and renal clearance $^{[6]}$. Hence, a significant amount of efforts have been made to identify molecules that activate the GLP-1 pathway with improved pharmacokinetic properties.

Current available GLP-1 mimetics encompass two classes of agents ${ }^{[7]}$ : GLP-1R agonists (eg, exenatide and liraglutide) that are structurally related to GLP-1 and orally available DPP-IV inhibitors (eg, sitagliptin, saxagliptin and vildagliptin) that elevate circulating GLP-1 concentrations via limiting the degradation of endogenous GLP-1. Various human and animal 
studies show that these therapeutic agents achieve meaningful reductions in hemoglobin $\mathrm{A}(1 \mathrm{c})$ (HbA1c) levels without causing significant hypoglycemia. In addition, decreases in body weight have been observed with GLP-1R agonists accompanied by beneficial effects on human cardiac function ${ }^{[8]}$. While the market outlook for GLP-1 mimetics seems favorable, certain limitations, such as repeated injections required for synthetic peptides and lack of weight loss following treatment with DPP-IV inhibitors, may prevent us from fully capturing the therapeutic potential of GLP-1 mimetics. Orally active, non-peptidic GLP-1R agonists thus remain a promising approach to tackle such challenges.

The GLP-1R belongs to the class B family of G-proteincoupled receptors (GPCRs), consisting of seven membrane spanning domains connected to each other by three extracellular and three intracellular loops. It is widely recognized that GPCRs represent the most important target class for therapeutic intervention ${ }^{[9]}$. Although there are a numerous examples of class A GPCRs being activated by small molecule agonists, very few non-peptidic ligands have been discovered for class B GPCRs. For instance, only several small molecule GLP-1R agonists with diverse structural features and functional selectivity (orthosteric, allosteric, ago-allosteric, inverse, or partial/ full agonists) were reported ${ }^{[10-15]}$. Among them, substituted cyclobutane Boc5 is the one that has been investigated most systematically both in vitro and in vivo to date.

\section{Pharmacology}

Boc5 was discovered following a high-throughput screening campaign against 48,160 small molecule compounds by use of a luciferase reporter assay in HEK293 cells stably expressing the rat GLP-1R gene linked to cAMP response element. Acute intraperitoneal (ip) and oral administration of Boc5 (0.1, $0.3,2$, and $3 \mathrm{mg}$ ) dose-dependently inhibited food intake in normal C57BL/6J mice, an effect that could be blocked by pretreatment with specific GLP-1R antagonist exendin(9-39). Daily injection of Boc5 (2 or $3 \mathrm{mg}$; 6 weeks) into $d b / d b$ mice reduced $\mathrm{HbA1c}$, improved glucose tolerance and lowered body weight in $d b / d b$ diabetic mice ${ }^{[11]}$. Encouraged by these initial results, we further characterized the pharmacological properties of Boc5 in both normal and $d b / d b$ mice with emphasis on glycemic control and weight loss. In addition to the beneficial effects on glycemic control, cumulative food intake and body weight, Boc5 was shown to be capable of amplifying glucose-dependent insulin secretion, increasing insulin sensitivity, reducing body fat mass, slowing gastric emptying and inducing satiety at higher doses beyond the therapeutic window ${ }^{[16]}$. Clearly, the anti-diabetic action exerted by Boc5 truly resembled that of the native peptide and the therapeutic effects could only be seen in diabetic $d b / d b$ mice but not in normal animals, implying a good safety profile (Tables S1 and S2).

Although the $d b / d b$ mouse represents a convenient model of diabetes, it does not fully simulate the pathogenesis of human T2DM and obesity. Therefore, we subsequently explored the therapeutic utility of Boc5 in a mouse model of diet-induced obesity (DIO), commonly used in efficacy assessment of new anti-diabetic agents. Guided by a pilot dose ranging study in male C57BL/6J mice where DIO was completely preventable through intermittent Boc5 administration (Figure S1), we designed and carried out a comprehensive investigation for this indication. Three times a week, not once daily, ip injections of Boc5 $(0.3,3$, and $1 \mathrm{mg})$ for 12 weeks resulted in typical dose-dependent responses in regulating food intake, adiposity, glucose homeostasis and insulin sensitivity ${ }^{[17]}$, similar to that reported previously in $d b / d b$ mice ${ }^{[11,16]}$. Of interest is our finding that Boc5 was able of normalizing pancreas $\beta$-cell mass and islet size through suppression of compensatory $\beta$-cell hyperplasia in DIO mice resistant to insulin actions, accompanied normalization of dyslipidemia, adipocytokines dysregulation, adipocyte malfunction and liver injury ${ }^{[17]}$.

In several in vitro (luciferase reporter ${ }^{[11]}$ and binding ${ }^{[11]}$ assays) and short-term in vivo (food intake ${ }^{[11]}$, gastric emptying $^{[16]}$ and stimulation of insulin secretion ${ }^{[16]}$ ) studies, where GLP-1 or exenatide were used as positive controls, maximally stimulating effects of Boc5 were similar in magnitude to that of the peptides, albeit the latter being approximately 3 to 4 orders of magnitude more potent. Although the action site(s) for the anorectic effect induced by peripheral administration of GLP-1R agonists are not clear, several lines of evidence suggest that the inhibition on food intake caused by exenatide and liraglutide are mediated via activation of GLP-1R expressed on sub-diaphragmatic vagal afferents, as well as in the brain ${ }^{[18]}$. We do not know at this stage whether Boc5 passes the blood brain barrier, our earlier work demonstrated that Boc5, at a dose of $6 \mathrm{mg}$ elicited conditioned taste aversion (CTA) in C57BL/6J mice ${ }^{[16]}$, implying the involvement of both peripheral and central GLP-1 signaling pathways.

While the anti-diabetic effects of Boc5 were observable by oral $^{[11,16]}$ and subcutaneous administration (Figure S2), the oral route required significantly higher doses to show efficacy. On the other hand, exogenous Boc5 had been shown to possess a markedly extended duration of action on several biological systems ${ }^{[11,16,17]}$. Hence, additional experiments were conducted to examine the pharmacokinetic properties of Boc5 in Sprague-Dawley (SD) rats and C57BL/6J mice, following a single dose of oral ( $p o$ ), ip, or intravenous (iv) administration. Samples were assayed for the prototype drug using HPLC, and mean plasma concentration-time curve and pharmacokinetic parameters were calculated (Figure S3, Table S3). One of the most striking features of the results was that the plasma drug level was hard to measure after a po dose of Boc5 in both rats $(20 \mathrm{mg} / \mathrm{kg}$ ) and mice $(250 \mathrm{mg} / \mathrm{kg})$. In contrast, the prototype drug could be readily detected after either ip or iv administration $(20 \mathrm{mg} / \mathrm{kg}$ for rats and $75 \mathrm{mg} / \mathrm{kg}$ for mice). The halflives $\left(T_{1 / 2}\right)$ for Boc5 in rats and mice were $35.4 \mathrm{~h}$ and $12.1 \mathrm{~h}$ following ip administration, and $41.7 \mathrm{~h}$ and $8.71 \mathrm{~h}$ following iv administration, respectively; and the bioavailabilities in rats and mice were $71.6 \%$ and $51.2 \%$, respectively when injected intraperitoneally (Table S3). Since plasma protein binding was moderate for Boc5 in mice $(36.2 \% \pm 5.5 \%$; Table S4), we presume that the lack of free molecule after oral administra- 

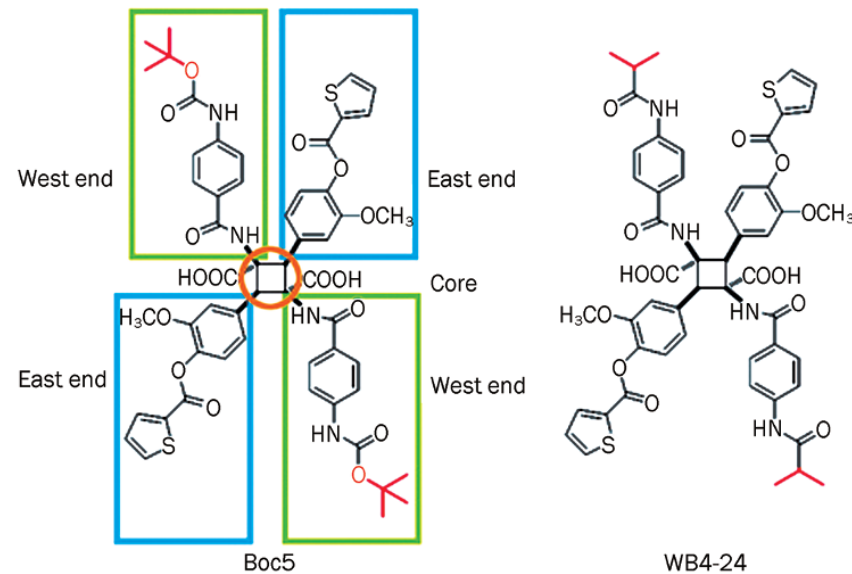

Figure 1. The structures of Boc5 and WB4-24.

tion may have been caused by metabolism in the gut, where the ester bonds at Boc (Figure 1, west end) and thienyl groups (Figure 1, east end) positions were quickly metabolized by esterase on the epithelial lining of the intestine (Figure S4). Further in vitro studies revealed that Boc5 was metabolized to 3 metabolites by B-type esterase(s) localized in the microsomal fraction of the mouse liver. These hydrolyzed metabolites (M-1, M-2, and M-3) were identified as dethiophenecarboxylBoc5, bisdethiophenecarboxyl-Boc5 and 2-thiophenecarboxylic acid, respectively, by use of UFLC-DAD-ESI-MS, with the help of authentic standard (Figure S4A). Luciferase reporter assay showed that dethiophenecarboxyl-Boc5 (M-1) at concentrations up to $100 \mu \mathrm{mol} / \mathrm{L}$ evoked maximally only $50 \%$ of Boc5 response (37\% of GLP-1 response) with an estimated $\mathrm{EC}_{50}$ value was $16.0 \mu \mathrm{mol} / \mathrm{L}$, approximately twelve times higher than that of Boc5 $(1.3 \mu \mathrm{mol} / \mathrm{L})$. Both bisdethiophenecarboxylBoc5 (M-2) and 2-thiophenecarboxylic acid (M-3) lost agonist activities (Figure S4B). In addition, Boc5 was very stable in hepatic microsomes from mini-pigs (PLM), rats (RLM) and humans (HLM), as well as human intestinal microsomes (HIM); the conventional metabolic enzymes in human including cytochrome P450s (CYPs), UDP-glucuronosyltransferases (UGTs) and esterases were not involved in the metabolism of this novel molecule (data not shown). These data were consistent with relatively long plasma half-lives of Boc5 after nonoral routes of administration.

\section{Improvement}

In order to understand the structure-activity relationship of this class of GLP-1R agonists, a series of symmetrical chemistry efforts were made to optimize the core, west and east ends of Boc5 (Figure 1). Among several dozen analogues prepared, WB4-24 demonstrated more potent bioactivities than Boc5 both in vitro (GLP-1R binding and cAMP response) and in vivo (food intake inhibition) ${ }^{[19]}$. With these results, we designed and performed an array of experiments using the mouse DIO model described above to characterize this compound. First, we studied the effects of WB4-24 on energy metabolism. As shown in Figures 2A and 2B, intermittent WB4-24 administration (ip, 3 times a week) led to a dosedependent and significant reduction in body weight over the first 12-week treatment period. The maximal weight losses were approximately $31.8 \%(P<0.01), 13.0 \%(P<0.01)$ and $8.3 \%$ $(P<0.05)$ at 3,1 , and $0.3 \mathrm{mg}$ on different time points $(4,6$, and 8 weeks, respectively) compared to that of obese controls. In addition, the pace of weight loss in $3 \mathrm{mg} \mathrm{WB4-24}$ treated DIO mice was much faster than that of Boc5, reaching to the lean control level within the first 4 weeks of therapy (Figure 2A). Likewise, subchronic WB4-24 administration dosedependently inhibited daily food intake, and $3 \mathrm{mg}$ of WB424 displayed better potency than Boc 5 between weeks 1 and 5 (Figures 2C and 2D).

Next, we investigated the effects of this derivative of Boc5 on blood glucose homeostasis. Glucose tolerance was quantified as the area-under-the-curve integrated from 0 120 min $\left(\mathrm{AUC}_{120}\right)$ after an intraperitoneal glucose challenge (intraperitoneal glucose tolerance test, IPGTT). Prior to initiating therapy with WB4-24, DIO mice showed impaired glucose tolerance relative to lean control mice ( $P<0.01$, Figures $3 \mathrm{~A}$ and $3 \mathrm{~F})$. IPGTTs conducted at 4 and 12 weeks after subchronic WB4-24 ip injections revealed a dose-dependent restoration of glucose tolerance, such that the glucose profile of mice receiving $3 \mathrm{mg}$ of WB4-24 was indistinguishable from that of lean controls at 4 weeks ( $P>0.05$, Figures 3B, 3C, and 3F). WB4-24 and Boc5 at 3 $\mathrm{mg}$ played similar roles in improving glucose tolerance during the 12-week observation period (Figures 3B and 3C).

Serum satiety hormone concentrations were also measured to study the endocrine status of treated animals. At the end of 12-week treatment, the blood samples were collected from WB4-24-treatd (3 mg), Boc5-treated (3 mg), lean and obese control groups. Fasting satiety hormones including insulin, leptin, active GLP-1 and glucagon levels were analyzed. Compared to lean controls, obese mice had significantly higher serum insulin $(P<0.01)$, leptin $(P<0.01)$ and glucagon $(P<0.01)$ concentrations with the exception of active GLP-1 $(P>0.05$, Figure 4). Such an adaptation of serum satiety hormones in the obese state has been documented previously ${ }^{[20]}$. Conversely, both WB4-24 and Boc5 treatment significantly reduced the serum insulin $(P<0.01)$, leptin $(P<0.01)$ and glucagon $(P<0.05)$ levels, which was in agreement with the reported studies on peptidic GLP-1R agonists ${ }^{[21]}$. Suppression of glucagon secretion following subchronic administration of Boc5 and WB4-24 further supports our dogma that this class of molecules truly activates the full spectrum of GLP-1 actions (Table 1).

Of note was our observation that each WB4-24 dose group markedly regained body weight, after a rapid initial fall, even during the treatment course (Figure 2A). This surprising phenomenon prompted us to perform a further study through reintroduction of $3 \mathrm{mg}$ WB4-24 to the same dose group in parallel with both lean and obese controls. Figure 2A shows that 8 weeks after the cessation of WB4-24 administration, mice became obese again with body weight rebound to the pretreatment level ( $\sim 40 \mathrm{~g})$; 8-week re-treatment only induced a moderate weight loss $(7.5 \%)$, approximately one-fourth of that 


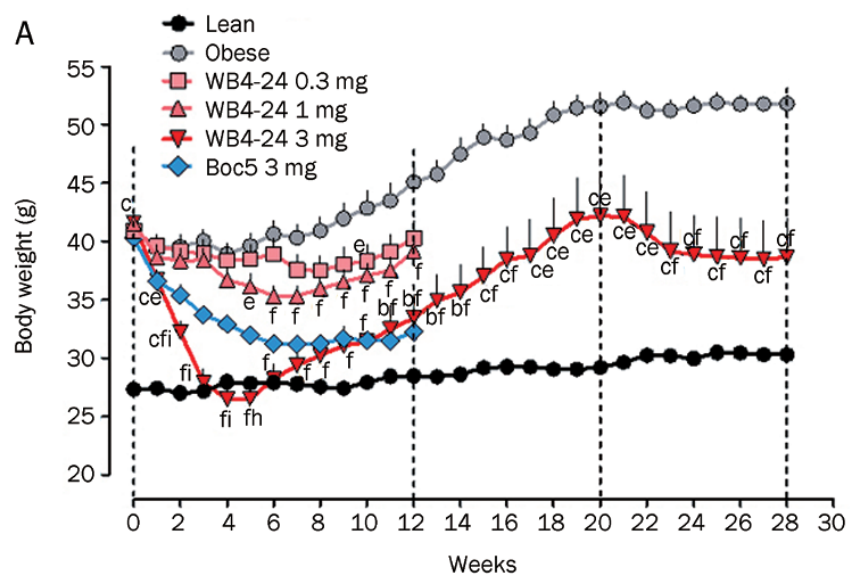

C

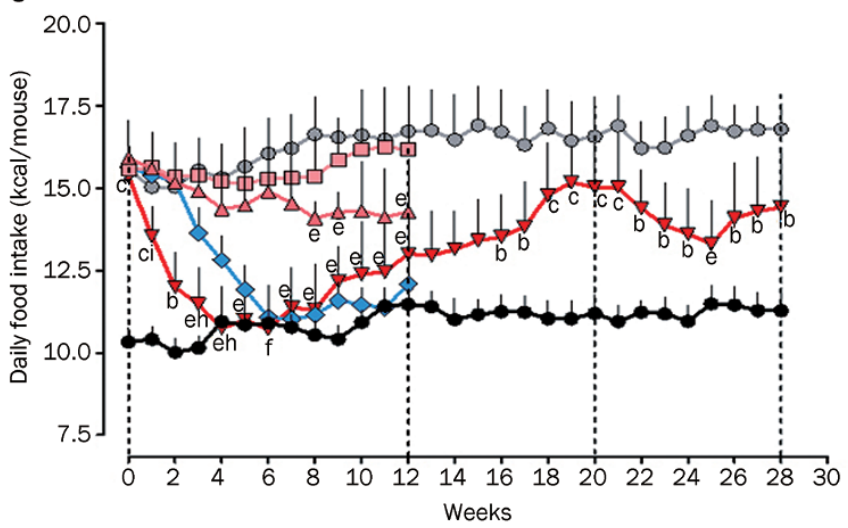

B

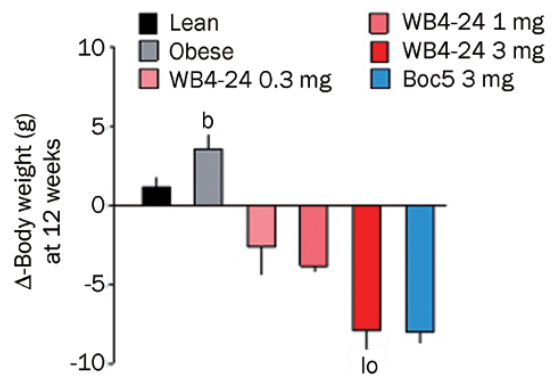

D

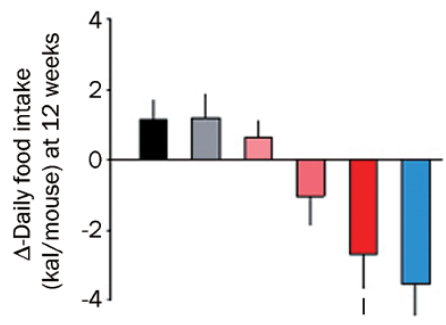

Figure 2. Effects of WB4-24 treatment on body weight and food intake in diet-induced obese (DIO) mice. Animals were maintained on high fat diet (HFD) for 12 weeks and then randomly assigned into 4 treatment groups with matched body weight. They were injected (ip), 3 times a week (Monday, Wednesday and Friday), with 0 (obese control), 0.3, 1, or $3 \mathrm{mg}$ of WB4-24 (1\% DMSO, 20\% PEG400 in saline, pH 7.4, $0.5 \mathrm{~mL}$ ) for $12 \mathrm{meeks.} \mathrm{To} \mathrm{compare}$ the in vivo effects of WB4-24 and Boc5, a positive control group treated with $3 \mathrm{mg}$ of Boc5, previously proven to be effective in combating obesity, was presented. A further comparator group of mice consuming standard chow diet (lean control) was used to index responses to normal values. Except for animals in $3 \mathrm{mg}$ WB4-24, obese and lean control groups, all mice were sacrificed at the end of therapy. The remaining mice were kept under observation without therapeutic intervention. An 8-week new treatment regimen (3 mg WB4-24) was introduced two months after the cessation of the initial therapy. Time course of body weight changes (A) and daily food intake (C) over the 28-week investigational period were monitored. The changes of body weight ( $\Delta$-body weight, B) and daily food intake ( $\Delta$-daily food intake, $D$ ) over the first 12-week period were analyzed. Statistical analysis was performed using GraphPad Prism software (GraphPad, San Diego, CA, USA) by one-way analysis of variance (ANOVA), followed by Bonferroni post hoc analysis. Values are presented as mean \pm SEM. $n=6-9$ per group. ${ }^{b} P<0.05,{ }^{\circ} P<0.01$ compared with lean controls. ${ }^{e} P<0.05,{ }^{f} P<0.01$ compared with obese controls. ${ }^{\mathrm{h}} P<0.05,{ }^{\mathrm{i}} P<0.01$ compared with $3 \mathrm{mg}$ Boc5-treated mice. ${ }^{\mathrm{I}} P<0.01$ compared with $0.3 \mathrm{mg}$ WB4-24-treated mice. ${ }^{\circ} P<0.05$ compared with $1 \mathrm{mg}$ WB4-24-treated mice.

Table 1. Comparison of the therapeutic effects of glucagon-like peptide-1 (GLP-1) and Boc5.

\begin{tabular}{lll}
\hline Type 2 diabetes mellitus & \multicolumn{1}{c}{ GLP-1 } \\
\hline Insulin secretion $\downarrow$ & Insulin secretion $\uparrow$ & $\sqrt{ }$ \\
Insulin resistance & Insulin sensitivity $\uparrow$ & $\sqrt{ }$ \\
$\beta$-cell function $\downarrow$ & B-cell function $\uparrow$ & $\sqrt{ }$ \\
Hyperglucagonemia & Glucagon secretion $\downarrow$ & $\sqrt{ }$ \\
Obesity & Body weight $\downarrow$, food intake $\downarrow$ & $\sqrt{ }$ \\
Gastric emptying $\uparrow,-$, or $\downarrow$ & Gastric emptying $\downarrow$ & $\sqrt{ }$ \\
Hyperlipidaemia & Triglyceride $\downarrow$, cholesterol $\downarrow$, nonesterified fatty acid $\downarrow$ \\
\hline
\end{tabular}



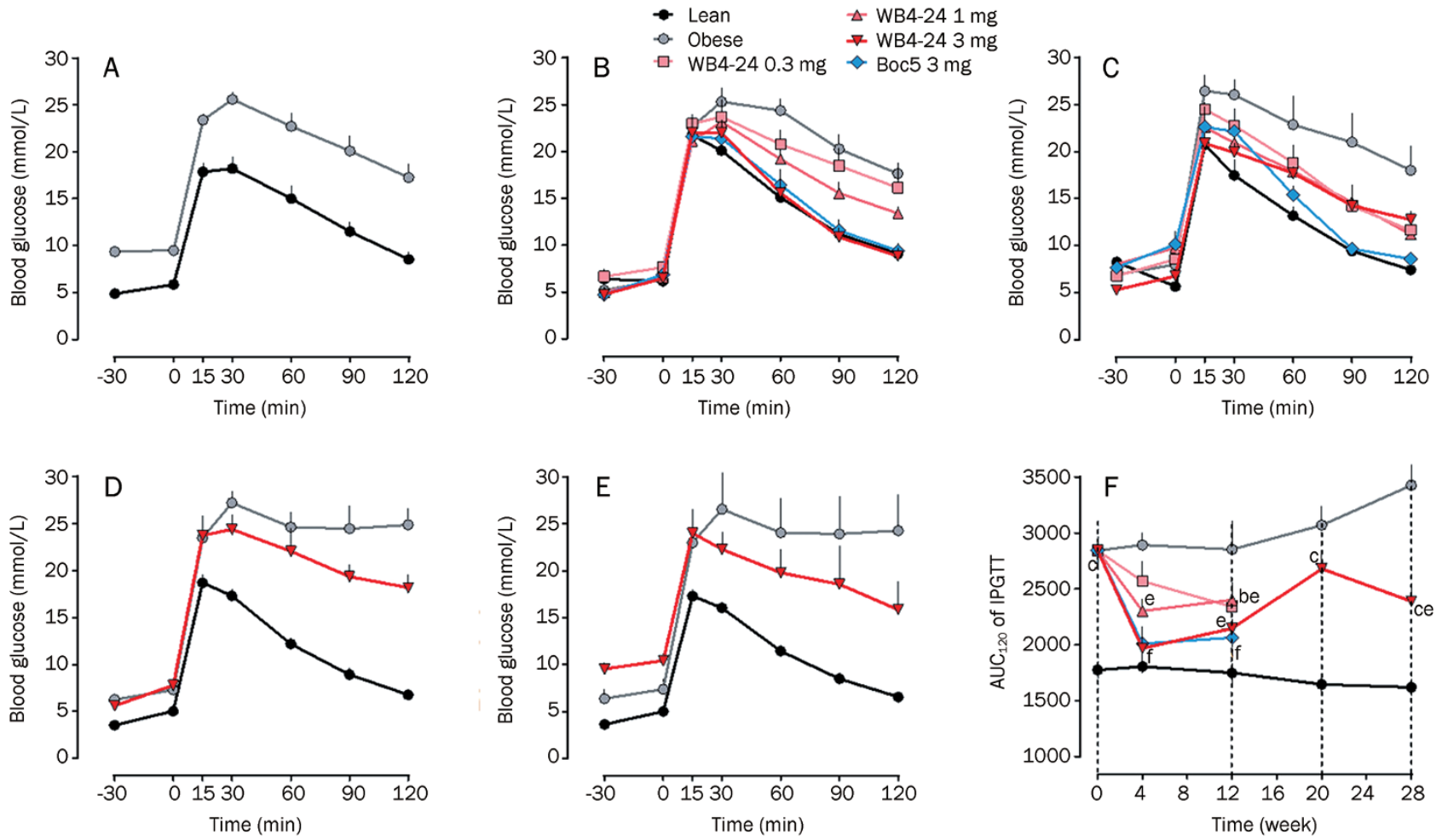

Figure 3. Effects of WB4-24 on glucose homeostasis in DIO mice. The experiments were conducted as described in Figure 1 legend and intraperitoneal glucose tolerance tests (IPGTTs) were carried out at week 0 (A), 4 (B), 12 (C), 20 (D), and 28 (E) after initial treatment with WB4-24. The glucose areaunder-curve integrated from 0-120 min $\left(\mathrm{AUC}_{120}\right)$ was calculated for each mouse $(\mathrm{F})$. Statistical analysis was performed using GraphPad Prism software by one-way analysis of ANOVA, followed by Bonferroni post hoc analysis. Values are presented as mean \pm SEM. $n=6-9$ per group. ${ }^{b} P<0.05,{ }^{c} P<0.01$ compared with lean controls. ${ }^{e} P<0.05,{ }^{f} P<0.01$ compared with obese controls.

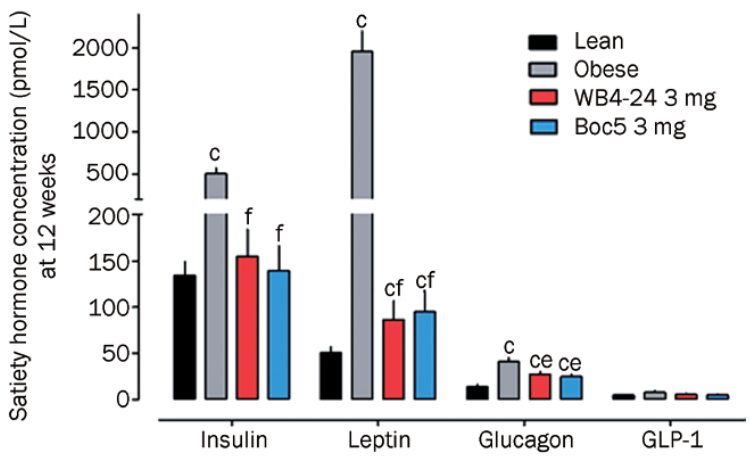

Figure 4. Effects of WB4-24 on serum satiety hormone concentrations in DIO mice. The experiments were conducted as described in Figure 1 legend. At the end of 12-week treatment, blood samples were collected from WB4-24-treated (3 mg), Boc5-treated (3 mg), lean and obese control groups. Fasting satiety hormones including insulin, leptin, active GLP-1 and glucagon levels were analyzed using Luminex 200 system (Luminex, Austin, USA) with the mouse endocrine MILLIPLEX MAP kit supplied by Millipore (Billerica, MA, USA). Statistical analysis was performed using GraphPad Prism software by one-way analysis of ANOVA, followed by Bonferroni post hoc analysis. Values are presented as mean \pm SEM. $n=6-9$ per group. ${ }^{\circ} P<0.01$ compared with lean controls; ${ }^{e} P<0.05,{ }^{f} P<0.01$ compared with obese controls.

produced by the initial treatment, accompanied by attenuated inhibitory action on food intake (Figure 2C) and failure to restore glucose tolerance to the normal state $(P<0.05$, Figures $3 \mathrm{D}, 3 \mathrm{E}$, and $3 \mathrm{~F}$ ).

It is possible that such a reduced efficacy may have resulted from potential immunogenicity of cyclobutane derivatives because of their relatively large molecular sizes $(>1000)$. The peptidic GLP-1 mimetic, exenatide, has been shown to elicit specific antibody responses in about $40 \%$ of patients ${ }^{[2,23]}$. In one study reported at EASD (European Association for the Study of Diabetes) 2010, antibody titers to exenatide reached a peak early in the treatment ${ }^{[24]}$, whereas in another study, $61 \%$ of patients developed antibody reactions to exenatide after 26-week treatment, and patients with high antibody titers had smaller $\mathrm{HbA1c}$ reductions than those with low titers ${ }^{[25]}$. This may provide a link between decreased hypoglycemic efficacy and patients with antibodies to exenatide. Therefore, it was prudent to investigate the possibility in the context of repeated treatments with WB4-24 (molecular weight=1074).

Serum samples were subsequently collected from mice that received the 2nd WB4-24 treatment $(3 \mathrm{mg})$ upon completion of dosing and specific antibodies were measured using an ELISA assay developed in-house. Briefly, WB4-24-carrier protein conjugate was prepared according to the 1-ethyl-3(3-dimethylaminopropyl) carbodiimide hydrochloride (EDC) coupling approach ${ }^{[26]}$. The molar ratio of WB4-24 to carrier protein (bovine serum albumin, BSA) was approximately 30:1, evaluated by spectroscopy and trinitrobenzene sulfonate 
(TNBS) method ${ }^{[27]}$. A 96-well microtiter plate was coated with $100 \mu \mathrm{l}(2 \mu \mathrm{g} / \mathrm{mL})$ of the conjugate at $4{ }^{\circ} \mathrm{C}$ overnight. The serum samples were serially diluted from 1:50 and added to each well of the blocked ( $20 \%$ normal sheep serum) plate. Bound antibody was then detected by goat anti-mouse IgHRP (BD Pharmingen, NJ, USA). Endpoint titers were finally determined at the $\mathrm{x}$-axis intercept of the dilution curve, at two times the absorbance $\left(A_{450}\right)$ given by the lean/obese control serum $^{[28]}$. The results showed that 4 of $6(67 \%)$ WB4-24-treated mice developed WB4-24 antibodies at titers of 23.38, 240.3, 27 and 487.1, respectively, while the titers of other two mice were too low to be measured. This range of anti-WB4-24 antibody titers was similar to that of anti-exenatide antibody $(\leq 125)$ in humans ${ }^{[29,30]}$. Statistical analysis (Figure 5) revealed that antibody titer had a positive correlation with the efficacy in terms of daily food intake in treated DIO mice $(r=0.8227 ; P<0.05)$. In the cases of body weight and $\mathrm{AUC}_{120}$ of IPGTT, the correlations were not statistically significant $(P>0.05)$. Therefore, this observation may be suggestive of the existence of an antibodymediated neutralizing mechanism, further studies are required to examine whether GLP-1R paralysis (down-regulation or tolerance) caused by continuous exposure to high doses of WB4-24 contributed to the reduced efficacy ${ }^{[31]}$. Moreover, it is of importance to note that the antibody formation may result in safety issues, immune-related and hypersensitivity reactions in particular, although such adverse events have yet to be unmasked in our hands ${ }^{[32]}$.

\section{Conclusion}

Obvious, limited success has been achieved since Boc5 was first reported in 2007 as a full non-peptidic agonist for GLP1R. Although WB4-24 exhibited more potent bioactivities than Boc5 at the same dose level, its bioavailability and exposure level in the plasma should be, in theory, comparable to Boc5 because both ester and thienyl groups on the east end remain unchanged (Figure 1). Our inability to develop a 'druggable' molecule beyond the current structural class is consistent with the inherent difficulties in discovery of non-peptidic ligands for class B GPCRs. The unique structural architecture and activation mechanism of GPCRs are pivotal factors in this issue. Little secondary structure information is available, and rational drug design has to rely on low homology template modeling of rhodopsin ${ }^{[33]}$. High resolution crystal structures of GPCRs will certainly expand our understanding of how a ligand gains access to and binds within the receptor binding pocket and finally guide the structure-based drug design ${ }^{[34]}$. It is encouraging that Underwood ${ }^{[35]}$ recently released the crystal structure of the extracellular domain of GLP-1R showing the molecular details of GLP-1 binding to the receptor extracellular N-terminal domain (ECD), an essential step in the two-domain binding mechanism applicable to class B GPCRs in general. The latest findings of Dezelak and Bavec ${ }^{[36]}$ suggested that the third intracellular loop of GLP-1R worked as competitive substrate for mono-ADP-ribosyltransferase, which reduced mono-ADP-ribosylation of $\beta$ subunit. This posttranslational modification of third loop of GLP-1R might represent a possible novel mechanism of receptor activity regulation and a potential target in treatment of T2DM. In conjunction with our determination of the crystal structures of Boc $5^{[19]}$ and WB424 (unpublished data), these latest developments will support us to characterize the binding site and ligand-mediated conformational changes induced by GLP-1, Boc5 or WB4-24, and to facilitate molecular modeling strategies to discover more potent and 'druggable' small molecule agonists.

\section{Acknowledgements}

We are indebted to Jing MA, Jing-kang SHEN, and Cai-hong ZHOU for valuable discussions, and to Dale E MAIS for critical review of this manuscript. This work was supported in part by grants from the Ministry of Science and Technology of China (2009ZX09302-001 and 2012ZX093004011), the Chinese Academy of Sciences (KSCX1-YW-02-2 and KSCX2-YWR-17), Shanghai Science and Technology Development Fund (074319114, 08DZ2291300, and 09DZ2291200), the K C Wong Education Foundation and the CAS-Novo Nordisk Research Fund.

\section{Author contribution}

Ming-wei WANG, Da-wei MA, Da-fang ZHONG, Chuan LI, and Ling YANG designed the research; Min HE, Ni GUAN,
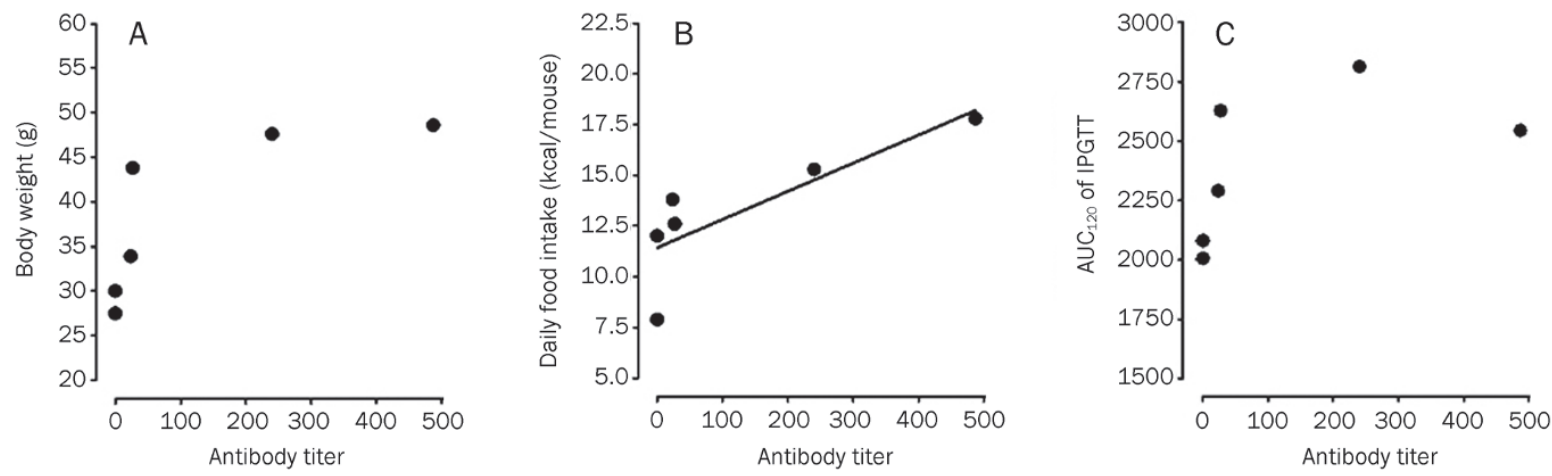

Figure 5. Correlation between plasma antibody titers and therapeutic efficacy in DIO mice treated with WB4-24. WB4-24 antibody titers had no statistically significant correlation with body weight ( $r=0.7758, P=0.698, n=6$; A) or AUC $_{120}$ of IPGTT ( $r=0.5627, P=0.245, n=6$; C). The line in column $B$ represents the positive correlation between WB4-24 antibody titers and daily food intake $(r=0.8227, P=0.0444, n=6)$. Statistical analysis was performed using GraphPad Prism software by Pearson's correlation analysis. 
Wei-wei GAO, Guang-bo GE, Qing LIU, Xiao-yan WU, and Xiao-yan CHEN performed the experiments; Ming-wei WANG, Da-fang ZHONG, Guang-bo GE, Chuan LI, and Ling YANG analyzed the data; and Min HE and Ming-wei WANG wrote the paper.

\section{References}

1 Report of the expert committee on the diagnosis and classification of diabetes mellitus. Diabetes Care 2003; 26 Suppl 1: S5-20.

2 Screening for Type 2 Diabetes. Report of a World Health Organization and International Diabetes Federation meeting. 2003. Available from: http://www.who.int/diabetes/publications/en/screening_ mnc03.pdf

3 Gallwitz B. Glucagon-like peptide-1-based therapies for the treatment of type 2 diabetes mellitus. Treat Endocrinol 2005; 4: 361-70.

4 Meier JJ, Nauck MA. The potential role of glucagon-like peptide 1 in diabetes. Curr Opin Investig Drugs 2004; 5: 402-10.

5 Baggio LL, Drucker DJ. Biology of incretins: GLP-1 and GIP. Gastroenterology 2007; 132: 2131-57.

6 Drucker DJ. Minireview: the glucagon-like peptides. Endocrinology 2001; 142: 521-7.

7 Drab SR. Incretin-based therapies for type 2 diabetes mellitus: current status and future prospects. Pharmacotherapy 2010; 30: 609-24.

8 Grieve DJ, Cassidy RS, Green BD. Emerging cardiovascular actions of the incretin hormone glucagon-like peptide-1: potential therapeutic benefits beyond glycaemic control? Br J Clin Pharmacol 2009; 57: 1340-51.

9 Eglen RM. GPCRs revisited: new insights lead to novel drugs. Pharmaceuticals 2011; 4: 244-72.

10 Knudsen LB, Kiel D, Teng M, Behrens C, Bhumralkar D, Kodra JT, et al. Small-molecule agonists for the glucagon-like peptide 1 receptor. Proc Natl Acad Sci U S A 2007; 104: 937-42.

11 Chen D, Liao J, Li N, Zhou C, Liu Q, Wang G, et al. A nonpeptidic agonist of glucagon-like peptide 1 receptors with efficacy in diabetic $d b / d b$ mice. Proc Natl Acad Sci U S A 2007; 104: 943-8.

12 Gong YD. A novel 3-(8-Chloro-6-(trifluoromethyl)imidazo[1,2-a] pyridine-2-yl)phenyl acetate skeleton and pharmacophore model as glucagon-like peptide 1 receptor agonists. Bull Korean Chem Soc 2010; 31: 3760-4.

13 Sloop KW, Willard FS, Brenner MB, Ficorilli J, Valasek K, Showalter $A D$, et al. Novel small molecule glucagon-like peptide-1 receptor agonist stimulates insulin secretion in rodents and from human islets. Diabetes 2010; 59: 3099-107.

14 Wang MW, Liu Q, Zhou CH. Non-peptidic glucagon-like peptide-1 receptor agonists: aftermath of a serendipitous discovery. Acta Pharmacol Sin 2010; 31: 1026-30.

15 Liao JY, Hong YF, Wang Y, Von geldern TW, Zhang KE. Phenylalanine derivatives and their use as non-peptide GLP-1 receptor modulators. WO Patent 2011094890. 2011; August 11.

16 Su H, He M, Li H, Liu Q, Wang J, Wang Y, et al. Boc5, a non-peptidic glucagon-like peptide-1 receptor agonist, invokes sustained glycemic control and weight loss in diabetic mice. PLoS ONE 2008; 3: e2892.

17 He M, Su H, Gao W, Johansson SM, Liu Q, Wu X, et al. Reversal of obesity and insulin resistance by a non-peptidic glucagon-like peptide-1 receptor agonist in diet-induced obese mice. PLOS ONE 2010; 5: e14205.

18 Kanoski SE, Fortin SM, Arnold M, Grill HJ, Hayes MR. Peripheral and central GLP-1 receptor populations mediate the anorectic effects of peripherally administered GLP-1 receptor agonists, liraglutide and exendin-4. Endocrinology 2011; 152: 3103-12.
19 Liu Q, Li N, Yuan YY, Lu HL, Wu XY, Zhou CH, et al. Cyclobutane derivatives as novel non-peptidic small molecule agonists of glucagonlike peptide-1 receptor. J Med Chem 2011. Doi: 10.1021/jm201150j.

20 Parnell JA. Differential secretion of satiety hormones with progression of obesity in JCR:LA-corpulent rats. Obesity 2008; 16: 736-42.

21 Toft-Nielson M, Madsbad S, Holst JJ. The effect of glucagon-like peptide 1 (GLP-1) on glucose elimination in healthy subjects depends on the pancreatic glucoregulatory hormones. Diabetes 1996; 45: 552-6.

22 Buse JB, Henry RR, Han J, Kim DD, Fineman MS, Baron AD. Effects of exenatide (exendin-4) on glycemic control over 30 weeks in sulfonylurea-treated patients with type 2 diabetes. Diabetes Care 2004; 27: 2628-35.

23 DeFronzo RA, Ratner RE, Han J, Kim DD, Fineman MS, Baron AD. Effects of exenatide (exendin-4) on glycemic control and weight over 30 weeks in metformin-treated patients with type 2 diabetes. Diabetes Care 2005; 28: 1092-100.

24 Fineman M. Antibodies to exenatide did not cross-react with human GLP-1 or glucagon or alter the efficacy or safety of exenatide. Diabetologia 2010; 53: S342.

25 Buse JB. The incidence of antibody formation and the levels of antibodies are lower with liraglutide than exenatide in a head-to-head comparison. Diabetologia 2010; 53: S341.

26 Hermanson GT. Preparation of hapten-carrier immunogen conjugates. In: Hermanson GT, editor. Bioconjugate Techniques. New York: Academic Press; 2008. p 745-82.

27 Singh KV, Kaur J, Varshney GC, Raje M, Suri CR. Synthesis and characterization of hapten-protein conjugates for antibody production against small molecules. Bioconjugate Chem 2004; 15: 168-73.

28 Hutchings CL, Gilbert SC, Hill AV, Moore AC. Novel protein and poxvirus-based vaccine combinations for simultaneous induction of humoral and cell-mediated immunity. J Immunol 2005; 175: 599606.

29 Kendall DM, Riddle MC, Rosenstock J, Zhuang D, Kim DD, Fineman MS, et al. Effects of exenatide (exendin-4) on glycemic control over 30 weeks in patients with type 2 diabetes treated with metformin and a sulfonylurea. Diabetes Care 2005; 28: 1083-91.

30 Zinman B, Hoogwerf BJ, Duran Garcia S, Milton DR, Giaconia JM, $\mathrm{Kim} \mathrm{DD}$, et al. The effect of adding exenatide to a thiazolidinedione in suboptimally controlled type 2 diabetes: a randomized trial. Ann Intern Med 2007; 146: 477-85.

31 Reidelberger RD, Haver AC, Apenteng BA, Anders KL, Steenson SM. Effects of exendin-4 alone and with peptide YY(3-36) on food intake and body weight in diet-induced obese rats. Obesity 2011; 19: 121-7.

32 Faludi P, Brodows R, Burger J, Ivanyi T, Braun DK. The effect of exenatide re-exposure on safety and efficacy. Peptides 2009; 30: 1771-4.

33 Stenkamp RE, Teller DC, Palczewski K. Crystal structure of rhodopsin: a G-protein-coupled receptor. ChemBioChem 2002; 3: 963-7.

34 Cherezov V, Rosenbaum DM, Hanson MA, Rasmussen SG, Thian FS, Kobilka TS, et al. High-resolution crystal structure of an engineered human beta2-adrenergic G protein-coupled receptor. Science 2007; 318: $1258-65$.

35 Underwood CR, Garibay P, Knudsen LB, Hastrup S, Peters GH, Rudolph $\mathrm{R}$, et al. Crystal structure of glucagon-like peptide-1 in complex with the extracellular domain of the glucagon-like peptide-1 receptor. J Biol Chem 2010; 285: 723-30.

36 Dezelak M, Bavec A. Third intracellular loop of glucagon like-peptide-1 receptor is coupled with endogenous mono-ADP-ribosyltransferase - novel type of receptor regulation? Eur J Pharmacol 2011; 666: $35-42$. 